\title{
Clinical nurse specialists in the catheter laboratory: a time for change or a bridge too far?
}

Purchasers and providers of health care in the United Kingdom are recognising extended roles for qualified nurses, in part related to the National Health Service reforms affecting junior doctors' training and hours of work.

Consultant cardiologists in Blackpool recognised nearly 20 years ago the need for nurse assistance during cardiac catheterisation to compensate for persistent shortages of junior medical staff. During this time all grades of qualified nurses based in the catheter laboratory have rotated as the assistant during cardiac catheterisation (transfemoral, brachial or radial routes) and coronary interventions. In addition, they routinely perform post-procedure groin compression and counsel patients before the procedure. No formal training has been available but they all have attended at least one coronary intervention course. Their role in coronary intervention continues to develop with consultant teaching and, as in several European centres, they are the "right hand assistant" for complex techniques such as rotational atherectomy.

Mean procedure times for mixed diagnostic and interventional lists performed with junior doctor or nurse assistance were recently determined as 33.5 and 29.6 minutes, respectively $-12 \%$ faster with nurse assistance.

Boulton et al, ${ }^{1}$ report in this issue their initial experience of extending the role of an experienced catheter laboratory nurse to primary operator for transfemoral cardiac catheterisation. They showed that in a short time an individual can be trained as a nurse specialist to perform elective procedures with equal safety and competence as a junior specialist registrar but with shorter screening and procedure times. The individual concerned had the essential close access to supportive consultant staff in case of emergency or complications. It should be remembered, however, that although the consultant is responsible for the overall care of the patient, nurses are responsible and accountable for all of their actions and omissions (professionally and legally). The supervising consultant and the employer must, therefore, be completely satisfied that the nurse specialist has the required experience, knowledge, and expertise. This should include skills in quick angiogram interpretation (particularly of left main disease where reduced contrast injections may be safer), venous cannulation, and the prescription and administration of intravenous drugs (within practice protocols). In addition, they must have a certificate in advanced life support. Patients will need to be aware that a nurse specialist will be performing the procedure and their consent obtained.

To assess the nurses' performance, records of cases including complications and radiation exposure should be available for peer review. A patient satisfaction survey would also be useful to support the development of the nurses' new role.

Nursing bodies have so far given cautious welcome to the development of clinical nurse specialists ${ }^{2}$ (the United Kingdom Central Council for Nursing, Midwifery and Health Visiting (UKCC) would prefer to call them advanced practitioners), but national leadership will be required to provide support, direction, and clarification of their role if advancement is to take place. By 1998 new programmes of education designed to meet UKCC requirements for clinical nurse specialists will be available throughout the United Kingdom. Perhaps it is an appropriate time to suggest that the British Cardiac Society consider supporting an advanced qualification in cardiac catheterisation for the nurse specialist (following a period of intense training of four months or more).

I acknowledge advice given by Susan Lee RGN BA(Hons) and Sue Arthur RGN.

Department of Cardiology,

DAVID HESKETH ROBERTS

Victoria Hospital NHS Trust,

Whinney Heys Road, Blackpool, UK

1 Boulton BD, Bashir Y, Ormerod OJM, Gribbin B, Forfar JC. Cardiac catheterisation performed by a clinical nurse specialist. Heart 1997;78: 194-7.

2 United Kingdom Central Council for Nursing, Midwifery and Health Visiting - transitional arrangements-specialist practitioner/specialist qualification. Register 1996;17:14-15. 